\title{
Genetic Analysis of Avirulence/Virulence of an Isolate of Magnaporthe grisea from a Rice Field in Texas
}

\author{
C. T. Chao and A. H. Ellingboe
}

First author: graduate research assistant, Plant Breeding and Plant Genetics Program and Department of Plant Pathology, University of Wisconsin, Madison 53706; second author: professor, Departments of Plant Pathology and Genetics, University of Wisconsin, Madison 53706. Current address of C. T. Chao: Department of Pomology, University of California, Davis 95616-8683.

Accepted for publication 18 September 1996.

\begin{abstract}
Chao, C. T., and Ellingboe, A. H. 1997. Genetic analysis of avirulence/virulence of an isolate of Magnaporthe grisea from a rice field in Texas. Phytopathology 87:71-76.

An isolate of Magnaporthe grisea, Tm4, from a rice field in Texas was crossed with a fertile laboratory strain, 70-6. The progenies showed segregation of avirulence/virulence on rice cvs. Newbonnet, Lemont, Lebonnet, Leah, and Katy. The avirulent/virulent segregation ratios were 29:6 on Newbonnet, Lemont, and Lebonnet; 28:7 on Leah; and 33:2 on Katy. There

certain strains had only one avirulence gene. Strains avirulent only on cv. Katy or only on cvs. Newbonnet, Lemont, and Lebonnet were test crossed with virulent siblings. Strains that gave progeny ratios approximating 1 avirulent: 1 virulent when crossed with virulent siblings were selected for further test crossing. Intercrosses between strains with possible single avirulence genes were made to determine whether these strains had the same or different avirulence genes. Many lines still segregated two genes for avirulence after three generations of backcrossing. This is based on the recovery of virulent progenies from crossing two avirulent siblings.
\end{abstract} was cosegregation on the first three cultivars. Several avirulent progenies were backcrossed to virulent parent 70-6. Three generations of backcrossing avirulent progenies to $70-6$ led to segregation ratios that suggested
Additional keywords: host-parasite interactions, rice blast disease.
Rice blast is one of the most severe fungal diseases on rice throughout the world and in the southern U.S. rice-growing area $(6,9)$. The disease is caused by Magnaporthe grisea (Hebert) Barr and usually is controlled by resistant cultivars and fungicides (7, 10). Resistant cultivars commonly do not stay resistant, however, due to the appearance of new virulent isolates (8). For practical plant breeding against blast disease in the southern United States, it is desirable to know the genetic control of avirulence/virulence in isolates obtained from U.S. rice fields. We wished to determine the number of genes in a field isolate that control specificity toward different cultivars and the types of interactions between these genes. This information should facilitate the identification of the individual resistance genes in the cultivars and the identification of new resistance genes.

In a previous study, we developed highly fertile laboratory strains that could mate with all siblings of opposite mating types and that were all pathogenic on rice (1). A cross was made between a fertile laboratory strain virulent on 11 U.S. rice cultivars and an isolate obtained from a rice field in Texas. Progenies avirulent on one or more rice cultivars were selected in each generation for further backcrosses to the laboratory strain. Test crosses also were made between the progenies to determine the genetic control of avirulence/virulence on several U.S. rice cultivars.

\section{MATERIALS AND METHODS}

Fungal strains. Isolate Tm4 was isolated from a rice field in Texas (12). Strain 70-6 was a highly fertile laboratory strain and virulent on 11 U.S. rice cultivars $(1,3)$. Progenies from a cross between Tm4 and 70-6 and their descendants are shown in Tables

Corresponding author: C. T. Chao; E-mail address: ctchao@ucdavis.edu

Publication no. P-1996-1104-02R

(C) 1997 The American Phytopathological Society
1 to 3. Strain 340-2 (genotype p11 P12 s11 s12) is avirulent on cv. Katy based on $P 12 s 12$ (3). $P$ is the avirulence locus, and $S$ is the corresponding suppressor locus. Strain 340-2 also is virulent on 10 other rice cultivars (K. Akutsu and A. H. Ellingboe, unpublished data). Strain 340-2 was test crossed with strains that had single genes for avirulence on cv. Katy (Fig. 1).

Mating and maintenance of the fungus. Mating was done by placing agar blocks of mycelium from two strains $\sim 4$ to $5 \mathrm{~cm}$ apart on oatmeal medium (4). The plates were held at room temperature $\left(20\right.$ to $\left.23^{\circ} \mathrm{C}\right)$ under fluorescent $(40 \mathrm{~W})$ and blue light $(360$ $\mathrm{mm}, 40 \mathrm{~W}$ ) for 2 to 3 weeks. Individual mature perithecia were transferred to complete media (11). Perithecia were crushed between two dissecting needles and dragged on the agar surface, and the process was observed under a $15 \times$ dissecting microscope. The procedure left a trail of asci. A fine glass needle was used to separate the mature asci from each other and from the perithecial debris. Asci with germinated ascospores were isolated after $24 \mathrm{~h}$ and transferred to oatmeal medium. Colonies with conidia were produced in 3 to 4 days. Conidia were spread onto a complete medium plate, and one germinated conidium was isolated from each colony. This procedure gave one progeny from each ascus. Strains prepared for storage were grown for 7 to 10 days on complete medium. The colony in agar was dried in a sterile hood for 3 days. The colony in dried agar was broken into pieces and stored in glassine paper bags at $-20^{\circ} \mathrm{C}$. Cultures stored using this method remained viable up to 6 years.

Rice cultivars. Seeds of U.S. rice cultivars were supplied by K. Moldenhauer, Rice Research and Extension Center, Stuttgart, AR (cvs. Newbonnet [PI 474580] and Katy [PI 527707]); D. M. Brandon, Rice Experimental Station, Biggs, CA (cv. S201 [CI 9974]); S. Linscomb, Louisiana Rice Research Station, Crowley (cv. Leah [CI 9979]); and M. A. Marchetti and M. S. Clung, USDA, Rice Research Station, Beaumont, TX (cvs. Lebonnet [CI 9882] and Lemont [PI 475833]). Cv. S201, which was susceptible to all strains, was used as a susceptible control in each inoculation. 
Inoculation. Several pieces of mycelium of each strain were transferred to oatmeal medium. After 10 days of growth, $25 \mathrm{ml}$ of distilled water containing $0.025 \%$ Tween 20 was poured onto the colonies. A bent needle was used to scrape the spores from the plates. Approximately $10^{6}$ to $10^{7}$ spores per ml were spray-inoculated with an atomizer onto the rice plants at the 2- to 3-leaf stage. The inoculated plants were covered with plastic bags, placed in a humidified chamber for $36 \mathrm{~h}$, and moved to a greenhouse bench at 23 to $26^{\circ} \mathrm{C}$. Lesions were first visible about 3 to 4 days after inoculation. Mature lesions appeared within 6 to 7 days. Avirulence/ virulence was usually scored 6 to 7 days after inoculation (3).

Scoring of avirulence/virulence. A 0 to 4 scale was used to score virulence (3). Infection type (IT) $0=$ no lesion; $1=$ minute brown to black flecks and spots; $2=2-$ to $3-\mathrm{mm}$ brown to black lesions; 3 = circular lesion with gray centers; and $4=$ large, diamond-shaped lesions with gray centers, commonly coalescing into long stripes. A clear separation of avirulent (IT 0-2) and virulent (IT 2-4) progeny strains could be made. The existence of only two clearly distinguishable IT ranges made classification of strains clear and unequivocal.

\section{RESULTS}

Segregation of avirulence/virulence in cross 85. Isolate Tm4 was avirulent on rice cvs. Newbonnet, Lemont, Lebonnet, Leah, and Katy and virulent on six other cultivars. Strain 70-6 was virulent on all 11 U.S. cultivars tested. Progenies from the cross between isolate Tm4 and strain 70-6 (cross 85) segregated for avirulence/virulence on cvs. Newbonnet, Lemont, Lebonnet, Leah, and Katy (Tables 1 to 3 ). No segregation occurred on the six cultivars on which both parent strains were virulent. Cross 85 had 29 avirulent and 6 virulent progenies on cvs. Newbonnet, Lemont, and Le-

TABLE 1. Cross number, parental strain number, infection types (IT), observed avirulence/virulence (avir/vir) segregation ratios for progenies, and chi-square values for one- and two-gene segregation ratios on rice cvs. Newbonnet, Lemont, and Lebonnet for progeny strains of Magnaporthe grisea originally derived from cross $85(\mathrm{Tm} 4 \times 70-6)$ and backcrosses to $70-6$

\begin{tabular}{|c|c|c|c|c|c|}
\hline \multirow[b]{2}{*}{ Cross no. } & \multirow[b]{2}{*}{ Parent no. } & \multirow[b]{2}{*}{ Parent IT $^{\mathrm{a}}$} & \multirow{2}{*}{$\begin{array}{c}\text { Progeny } \\
\text { avir/vir } \\
\text { ratio }\end{array}$} & \multicolumn{2}{|c|}{$\chi^{2 \mathrm{~b}}$} \\
\hline & & & & $1: 1$ & $3: 1$ \\
\hline 85 & Tm4 & 0 & & & \\
\hline & $70-6$ & $2-4$ & $29: 6$ & $15.11 * *$ & 1.15 \\
\hline
\end{tabular}

Cross 85 progenies backcrossed to 70-6 (BC1)

$\begin{array}{llllrl}87 & 85-15 & 0 & 12: 3 & 5.40 * & 0.20 \\ 88 & 85-28 & 0 & 20: 7 & 6.26 * & 0.01 \\ 89 & 85-54 & 0 & 20: 6 & 7.54 * * & 0.05 \\ 94 & 85-2 & 0 & 20: 3 & 12.57 * * & 1.75\end{array}$

Cross 88 progenies backcrossed to 70-6 (BC2)

$\begin{array}{llllll}221 & 88-1 & 0 & 9: 3 & 3.00 & 0.00 \\ 222 & 88-3 & 0-1 & 19: 5 & 8.17^{* *} & 0.22 \\ 223 & 88-20 & 0-1 & 15: 13 & 0.14 & 6.86^{* *} \\ 224 & 88-22 & 0-1 & 13: 10 & 0.39 & 4.19^{*} \\ 225 & 88-26 & 0 & 10: 9 & 0.05 & 5.07^{*} \\ 226 & 88-28 & 0 & 15: 1 & 12.25^{* *} & 3.00 \\ 127 & 88-10 & 0-1 & 11: 9 & 0.20 & 4.27^{*}\end{array}$

Cross 226 progenies backcrossed to $70-6$ (BC3)

\begin{tabular}{llrrrc}
272 & $226-9$ & $0-1$ & $20: 10$ & 3.33 & 1.11 \\
273 & $226-10$ & $2-4$ & $0: 39$ & $\ldots$ & $\ldots$ \\
274 & $226-11$ & $0-1$ & $20: 20$ & 0.00 & $13.33^{* *}$ \\
275 & $226-12$ & $1-2$ & $42: 25$ & $4.31^{*}$ & $5.42^{*}$ \\
276 & $226-15$ & $0-1$ & $16: 19$ & 0.26 & $16.01^{* *}$ \\
\hline
\end{tabular}

a Pathogenicity was scored on a 0 to 4 scale. ITs ranging from 0 to 2 were considered avirulent, and ITs ranging from 2 to 4 were considered virulent.

b $*$ indicates $P<0.05$; ** indicates $P<0.01$. bonnet (Table 1); 28 avirulent and 7 virulent progenies on cv. Leah (Table 2); and 33 avirulent and 2 virulent progenies on cv. Katy (Table 3). These segregation ratios indicate there are two genes that control avirulence on cvs. Newbonnet, Lemont, Lebonnet, and Leah. There was cosegregation for avirulence/virulence on cvs. Newbonnet, Lemont, and Lebonnet. The segregation ratio indicates two or more genes control avirulence on cv. Katy.

Segregation of avirulence/virulence on cvs. Newbonnet, Lemont, and Lebonnet in backcrosses. The segregation ratios of avirulence/virulence on Newbonnet, Lemont, and Lebonnet in the first backcross (BC1) to isolate 70-6 were 12:3, 20:7, 20:6, and $20: 3$ in crosses $87,88,89$, and 94 , respectively (Table 1 ). These ratios indicate that each progeny from cross 85 possesses two or more genes that control interactions on these cultivars.

Seven progenies from cross 88 (88-1, 88-3, 88-20, 88-22, 88-26, 88-28, and 88-10) were backcrossed again to strain 70-6 (crosses 221, 222, 223, 224, 225, 226, and 127) (backcross 2 [BC2]) (Table 1). All seven progenies from cross 88 were avirulent on cvs. Newbonnet, Lemont, Lebonnet, and Katy. The segregation ratios of avirulence/virulence in crosses 221, 222, and 226 were 9:3, 19:5, and 15:1, respectively, which indicates the segregation of two avirulence genes for cvs. Newbonnet, Lemont, and Lebonnet (Table 1). The segregation ratios of avirulence/virulence in crosses 223 , 224,225 , and 127 were $15: 13,13: 10,10: 9$, and 11:9, respectively (approximately 1:1), indicating a single avirulence gene most likely segregated in each of these crosses (Table 1). Five progenies from cross 226 (226-9, 226-10, 226-11, 226-12, and 226-15) were backcrossed again to 70-6 (backcross 3 [BC3]). One of the progenies, 226-10, was virulent on cvs. Newbonnet, Lemont, and Lebonnet and, when crossed with 70-6 (cross 273), gave all virulent progenies on these cultivars (Table 1). Three progenies, 226-9, 226-11,

TABLE 2. Cross number, parental strain number, infection type (IT), observed avirulence/virulence (avir/vir) segregation ratios for progenies, and chi-square values for one- and two-gene segregation ratios on rice cv. Leah for progeny strains of Magnaporthe grisea originally derived from cross 85 (Tm4 $\times$ 70-6) and backcrosses to $70-6$

\begin{tabular}{|c|c|c|c|c|c|}
\hline \multirow[b]{2}{*}{ Cross no. } & \multirow[b]{2}{*}{ Parent no. } & \multirow[b]{2}{*}{ Parent IT $^{\mathrm{a}}$} & \multirow{2}{*}{$\begin{array}{l}\text { Progeny } \\
\text { avir/vir } \\
\text { ratio }\end{array}$} & \multicolumn{2}{|c|}{$\chi^{2 b}$} \\
\hline & & & & $1: 1$ & $3: 1$ \\
\hline \multirow[t]{3}{*}{85} & Tm4 & 0 & & & \\
\hline & & & $28: 7$ & $12.60 * *$ & 0.47 \\
\hline & $70-6$ & $2-4$ & & & \\
\hline \multicolumn{6}{|c|}{$\begin{array}{l}\text { Cross } 85 \text { progenies backcrossed to } \\
70-6(\mathrm{BC} 1)\end{array}$} \\
\hline 87 & $85-15$ & 0 & $8: 7$ & 0.07 & 3.76 \\
\hline 88 & $85-28$ & 0 & $16: 11$ & 0.93 & 3.75 \\
\hline 89 & $85-54$ & 0 & $12: 14$ & 0.15 & $11.54 * *$ \\
\hline 94 & $85-2$ & 0 & $14: 9$ & 1.09 & 2.45 \\
\hline
\end{tabular}

Cross 88 progenies backcrossed to 70-6 (BC2)

\begin{tabular}{lllccc}
221 & $88-1$ & 0 & $6: 6$ & 0.00 & $4.00^{*}$ \\
222 & $88-3$ & $3-4$ & $0: 24$ & $\ldots$ & $\ldots$ \\
223 & $88-20$ & $0-2$ & $14: 14$ & 0.00 & $9.33^{* *}$ \\
224 & $88-22$ & $2-3$ & $0: 23$ & $\ldots$ & $\ldots$ \\
225 & $88-26$ & 0 & $10: 9$ & 0.05 & $5.07^{*}$ \\
226 & $88-28$ & $0-2$ & $10: 6$ & 1.00 & 1.33 \\
127 & $88-10$ & $3-4$ & $0: 26$ & $\ldots$ & $\ldots$ \\
oss 226 progenies backcrossed to & & & $\ldots$ \\
$70-6$ (BC3) & & & & & $\ldots$ \\
272 & $226-9$ & $2-4$ & $0: 30$ & $\ldots$ & $\ldots$ \\
273 & $226-10$ & $2-4$ & $0: 39$ & $\ldots$ & $\ldots$ \\
274 & $226-11$ & $0-1$ & $22: 18$ & 0.40 & $\ldots$ \\
275 & $226-12$ & $2-4$ & $10: 57^{*}$ & $32.97 * *$ & $128.96^{* *}$ \\
276 & $226-15$ & $2-4$ & $0: 35$ & $\ldots$ & $\ldots$ \\
\hline
\end{tabular}

a Pathogenicity was scored on a 0 to 4 scale. ITs ranging from 0 to 2 were considered avirulent, and ITs ranging from 2 to 4 were considered virulent.

b * indicates $P<0.05$; ** indicates $P<0.01$.

c A 10:57 ratio may indicate the presence of suppressor or other genes involved. 
and 226-15, were avirulent on Newbonnet, Lemont, and Lebonnet and, when crossed with 70-6 (crosses 272, 274, and 276), gave avirulent/virulent segregation ratios that approximated the 1:1 ratio expected if a single avirulence gene segregated in each cross. Cross 275 (226-12 x 70-6) gave a segregation ratio, 45:25, that fit neither a 1:1 nor a 3:1 ratio.

Segregation of avirulence/virulence on cv. Leah in backcrosses. The segregation ratios of avirulence/virulence on cv. Leah in BC1 were $8: 7,16: 11,12: 14$, and $14: 9$ in crosses $87,88,89$, and 94, respectively (Table 2). These ratios indicate that a single gene in each of these four crosses controls the interaction with cv. Leah. Eight progenies of cross 88 were backcrossed to strain 70-6. Four strains (88-1, 88-20, 88-26, and 88-28) were avirulent, and three strains (88-3, 88-22, and 88-10) were virulent on cv. Leah (Table 2).

Progenies from backcrossing three virulent strains (88-3, 88-22, and 88-10) to strain 70-6 (also virulent on cv. Leah) were all virulent on Leah. No evidence for the segregation of an avirulence gene was observed in crosses 222, 224, and 127. Strains 88-1, 88-20, 88-26, and 88-28 were avirulent on cv. Leah. When each strain was backcrossed to 70-6 (crosses 221, 223, 225, and 226), the progenies segregated in ratios of approximately 1 avirulent: 1 virulent on cv. Leah. Therefore, the evidence indicates that only one gene for avirulence on Leah segregated in each of the crosses 221, 223,225 , and 226. Four of the cross 226 progenies (226-9, 226$10,226-12$, and 226-15) that were virulent on Leah, were backcrossed to 70-6. Three of the backcrosses gave all virulent progenies $(272,273$, and 276). These results indicate that there is no avirulence gene segregating in crosses 272,273 , and 276 for reaction on Leah. Cross $275(226-12 \times 70-6)$ gave 10 avirulent and 57 virulent progenies on Leah. Strains 226-12 and 70-6 were both virulent on Leah, but avirulent progenies were recovered from a cross between the two. This result indicates that 262-12 has a gene

TABLE 3. Cross number, parental strain number, infection type (IT), observed avirulence/virulence (avir/vir) segregation ratios for progenies, and chi-square values for one- and two-gene segregation ratios on rice cv. Katy for progeny strains of Magnaporthe grisea originally derived from cross 85 (Tm4 $\times 70-6)$ and backcrosses to 70-6

\begin{tabular}{|c|c|c|c|c|c|}
\hline \multirow[b]{2}{*}{ Cross no. } & \multirow[b]{2}{*}{ Parent no. } & \multirow[b]{2}{*}{ Parent $\mathrm{IT}^{\mathrm{a}}$} & \multirow{2}{*}{$\begin{array}{c}\text { Progeny } \\
\text { avir/vir } \\
\text { ratio }\end{array}$} & \multicolumn{2}{|c|}{$\chi^{2 b}$} \\
\hline & & & & $1: 1$ & $3: 1$ \\
\hline \multirow[t]{2}{*}{85} & $\mathrm{Tm} 4$ & 0 & & & \\
\hline & $70-6$ & $2-4$ & $33: 2$ & $27.46 * *$ & 6.94 \\
\hline
\end{tabular}

Cross 85 progenies backcrossed to 70-6 (BC1)

$\begin{array}{llllll}87 & 85-15 & 0 & 11: 4 & 3.27 & 0.02 \\ 88 & 85-28 & 0-1 & 24: 3 & 16.33 * * & 2.78 \\ 89 & 85-54 & 0 & 22: 4 & 12.46 * * & 1.28 \\ 94 & 85-2 & 0 & 16: 7 & 3.52 & 0.36\end{array}$

Cross 88 progenies backcrossed to 70-6 (BC2)

$\begin{array}{lllrrl}221 & 88-1 & 0-1 & 9: 3 & 3.00 & 0.00 \\ 222 & 88-3 & 0 & 23: 1 & 20.17 * * & 5.56^{*} \\ 223 & 88-20 & 0-1 & 21: 6 & 8.33^{* *} & 0.11 \\ 224 & 88-22 & 0-1 & 20: 3 & 12.57 * * & 1.75 \\ 225 & 88-26 & 0 & 15: 4 & 6.37 * & 0.16 \\ 226 & 88-28 & 0-1 & 14: 2 & 9.00^{* *} & 1.33 \\ 127 & 88-10 & 0-1 & 26: 0 & \ldots & \ldots\end{array}$

Cross 226 progenies backcrossed to $70-6$ (BC3)

\begin{tabular}{llllll}
272 & $226-9$ & $1-2$ & $16: 14$ & 0.13 & $7.51^{* *}$ \\
273 & $226-10$ & $0-2$ & $16: 23$ & 1.26 & $24.01^{* *}$ \\
274 & $226-11$ & $1-2$ & $21: 19$ & 0.10 & $10.80^{* *}$ \\
275 & $226-12$ & $0-1$ & $56: 11$ & $30.22^{* *}$ & 2.63 \\
276 & $226-15$ & $0-2$ & $20: 15$ & 0.71 & $5.95^{*}$ \\
\hline
\end{tabular}

a Pathogenicity was scored on a 0 to 4 scale. ITs ranging from 0 to 2 were considered avirulent, and ITs ranging from 2 to 4 were considered virulent.

b * indicates $P<0.05$; ** indicates $P<0.01$. for avirulence on cv. Leah and a second gene that may suppress avirulence. Strain 226-11 was avirulent on Leah and, when crossed with 70-6 (cross 274), gave progenies that segregated in a 1 avirulent: 1 virulent ratio on Leah. This result indicates a single avirulence gene segregated in this cross.

Segregation of avirulence/virulence on cv. Katy in backcrosses. The segregation ratios of avirulence/virulence on cv. Katy in BC1 was $11: 4,24: 3,22: 4$, and 16:7 in crosses $87,88,89$, and 94, respectively (Table 3 ). These ratios indicate that two or more genes control avirulence on Katy in each of these four crosses.

The seven cross 88 progenies that were backcrossed again to 70-6 (BC2) were all avirulent on cv. Katy (Table 3). The observed avirulence/virulence segregation ratios in crosses 221, 222, 223, 224,225 , and 226 were consistent with the expected ratios if two or more avirulence genes were segregating in each cross. Cross $127(88-10 \times 70-6)$ had no virulent progenies. If $88-10$ contains several independent avirulence genes, it was possible that a sample size of 26 was not large enough to detect the rare virulent progenies. Five progenies (226-9, 226-10, 226-11, 226-12, and 226-15) from cross 226 were backcrossed again to 70-6 (Table 3, BC3). Four of the crosses $(272,273,274$, and 276) gave progenies that segregated in approximately 1 avirulent: 1 virulent ratios. Progenies 226-9, 226-10, 226-11, and 226-15 most likely contain a single gene for avirulence on cv. Katy. In cross 275 (226-12 $\times 70-6)$, the observed avirulent/virulent segregation ratio was 56:11, which indicates two or more avirulence genes segregated in this cross. Strain 226-12 probably possesses two or more avirulence genes for interaction with cv. Katy.

Establishment of strains with single avirulence genes. The results given in Tables 1 to 3 indicate that several genes control interactions with the five rice cultivars tested. There was cosegregation for avirulence/virulence on cvs. Newbonnet, Lemont, and Lebonnet and independent segregation for avirulence/virulence on cvs. Leah and Katy. Because 70-6 is virulent on all these cultivars, the assumption is that all of the avirulence genes have their origin in Tm4. Backcrossing to 70-6 for two to three generations has led to segregation ratios that indicate single avirulence genes are present in many $\mathrm{BC} 2$ or $\mathrm{BC} 3$ progenies.

If segregation occurs at only one avirulence locus, then intercrossings between siblings, one is virulent and one is avirulent on cvs. Newbonnet, Lemont, and Lebonnet (crosses 282, 286, and 288, respectively), should give single-gene segregation ratios (Table 4). Crosses 286 and 288 are consistent with single-gene segregation; however, cross 282 is not. The segregation in cross 282 is consistent with a 3:1 ratio, indicating two-gene segregation. Cross 272 segregated 20 avirulent:10 virulent on these three cultivars (Table 1), so the recovery of strain 272-16 with two genes for avirulence was expected. Crosses between siblings virulent on cvs. Newbonnet, Lemont, and Lebonnet (crosses 289 and 290) produced no avirulent but did produce virulent progenies as expected.

Of eight crosses $(292,293,294,295,296,298,299$, and 300) involving two strains, one was virulent and one was avirulent on

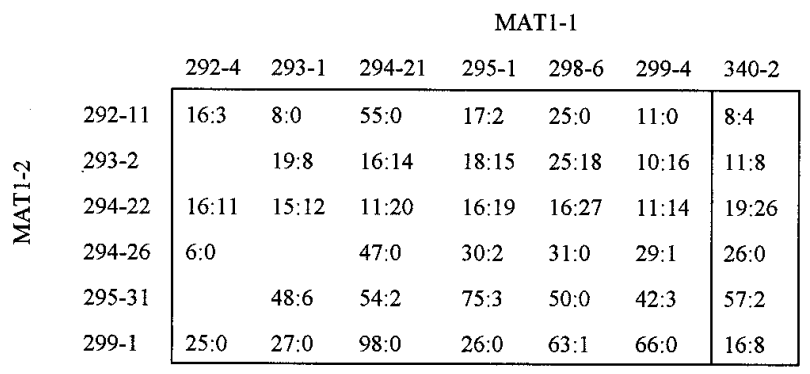

Fig. 1. Segregation for avirulence versus virulence of the progenies from crosses between strains of Magnaporthe grisea presumed to have single genes for avirulence on rice cv. Katy and from crosses between these strains and strain 340-2 (genotype p11 P12 s11 s12, avirulent on Katy). 
cv. Katy (Table 4), six crosses gave segregation ratios that approximated 1 avirulent: 1 virulent, and two crosses (296 and 300) gave ratios consistent with the segregation of two genes controlling avirulence on Katy. The segregation data given in Table 4 indicate that after several backcrosses to virulent strain 70-6, many crosses now segregate for only one avirulence gene.

Crosses between strains that each have a presumptive single avirulence gene for interaction with cvs. Newbonnet, Lemont, and Lebonnet. Strain 272-16 was crossed with strains 272-23 (cross 283) and 272-29 (cross 284) (Table 4). All three strains were avirulent only on cvs. Newbonnet, Lemont, and Lebonnet and were virulent on the other cultivars tested. These crosses gave all avirulent progenies on cvs. Newbonnet, Lemont, and Lebonnet. These results indicate that strains 272-16, 272-23, and 272-29 have an avirulence gene in common that is specific to cvs. Newbonnet, Lemont, and Lebonnet.

Strain 272-16 also was crossed with strain 229-1 (cross 287). Strain 229-1 was virulent on cv. Leah and avirulent on cvs. Newbonnet, Lemont, Lebonnet, and Katy. The cross gave 36 progenies. All were avirulent on cvs. Newbonnet, Lemont, and Lebonnet; 20 were avirulent and 16 were virulent on cv. Katy; and all were virulent on cv. Leah. These results indicate that the same avirulence gene specific to cvs. Newbonnet, Lemont, and Lebonnet is present in both 272-16 and 229-1. A single avirulence gene specific to cv. Katy from 229-1 segregated in the cross. There was no avirulence gene that was specific for cv. Leah in the cross.

Crosses between strains with presumptive single avirulence genes for interaction with cv. Katy. Crosses were made between strains with presumptive single avirulence genes to determine whether these strains had the same gene or different genes for avirulence on cv. Katy. If the progenies of cross 292 segregated for only one gene, then crosses between avirulent progenies should give all avirulent progenies. If cross 292 segregated for two avirulence genes, then some intercrosses between avirulent progenies should have produced virulent progenies. At least one avirulent progeny of each mating type from six crosses (292, 293, 294, 295, 298, and 299) (Table 4) was intercrossed in almost all possible combinations. The results of the crosses are shown in Figure 1.

There were three types of segregation ratios observed in these crosses. In the first type, all progenies from the cross were avirulent on cv. Katy. For example, when strain 292-11 was crossed with strains 293-1, 294-21, 298-6, and 299-4, all progenies were avirulent on Katy. These results are consistent with the four strains, 293-1, 294-21, 298-6, and 299-4, having one or more avirulence genes in common with 292-11. The second type of cross yielded few virulent progenies. For example, when strain 295-31 was crossed with 293-1, 294-21, 295-1, and 299-4, the crosses gave a low incidence of progenies that were virulent on Katy. These results indicate that the gene for avirulence in 295-31 is different from the genes in the other four strains (including its sibling 295-1) but that there is a tight linkage between the avirulence genes. Cross 295 , therefore, segregated for two avirulence genes, not one avirulence gene as previously suggested (Table 4). The third type of cross was illustrated in the crosses between 294-22 and six strains of opposite mating type. In these six crosses, the segregations most closely approximated a 1:1 ratio for avirulence/virulence on cv. Katy. In five of the six crosses, there were more virulent progenies than avirulent progenies.

Crosses between strains with presumptive single genes for interactions with cv. Katy and strain 340-2. When the six MAT1-2 avirulent strains were crossed with avirulent strain 340-2 (genotype 111 P12 s11 s12) (Fig. 1), with crosses involving strains 292$11,293-2$, and $299-1$, the progenies segregated in ratios of $8: 4$, $11: 8$, and 16:8 for avirulence/virulence on cv. Katy $\left(\chi_{3: 1}^{2}=0.44\right.$, 2.96 , and 0.89 , respectively). These results indicate two avirulence genes segregated in these crosses, one from strain 340-2 and one each from strains 292-11, 293-2, and 299-1. When strain 29422 was crossed with $340-2$, it gave 19 avirulent and 26 virulent progenies $\left(\chi_{7: 9}^{2}=0.04\right.$ and $\left.\chi_{1: 1}^{2}=1.09\right)$. This result indicates the segregation of two avirulence genes and two suppressors or two avirulence genes. When strains 294-26 and 295-31 were crossed with $340-2$, they gave avirulence/virulence segregation ratios of 26:0 and 57:2, respectively. These results indicate that strain 294-

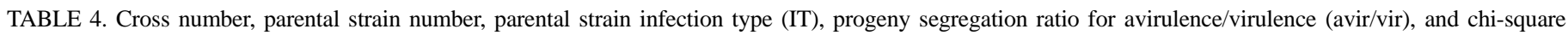

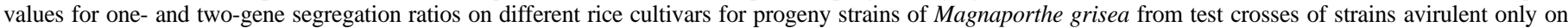
cvs. Newbonnet, Lemont, and Lebonnet and strains avirulent only on cv. Katy with virulent strains

\begin{tabular}{|c|c|c|c|c|c|c|c|}
\hline \multirow[b]{2}{*}{ Cross no. } & \multirow[b]{2}{*}{ Parent 1} & \multirow[b]{2}{*}{ Parent 2} & \multirow[b]{2}{*}{ Parent $1 \mathrm{IT}^{\mathrm{a}}$} & \multirow[b]{2}{*}{ Parent 2 IT } & \multirow[b]{2}{*}{ Avir/vir } & \multicolumn{2}{|c|}{$\chi^{2 \mathrm{~b}}$} \\
\hline & & & & & & $1: 1$ & $3: 1$ \\
\hline \multicolumn{8}{|c|}{ Segregation on } \\
\hline \multicolumn{8}{|c|}{ Cvs. Newbonnet, Lemont, and Lebonnet } \\
\hline 282 & $272-16$ & $272-21$ & 0 & $2-4$ & $34: 18$ & $4.92 *$ & 2.56 \\
\hline 286 & $272-11$ & $272-23$ & $2-4$ & $0-1$ & $21: 14$ & 1.40 & $4.20 *$ \\
\hline 288 & $272-11$ & $272-29$ & $2-4$ & 0 & $12: 12$ & 0.00 & $8.00 * *$ \\
\hline 283 & $272-16$ & $272-23$ & 0 & $0-1$ & $33: 0$ & $\ldots$ & $\ldots$ \\
\hline 284 & $272-16$ & $272-29$ & 0 & 0 & $21: 0$ & $\ldots$ & $\ldots$ \\
\hline 291 & $272-11$ & $229-1^{\mathrm{c}}$ & $2-4$ & 0 & $31: 27$ & 0.28 & $14.37 * *$ \\
\hline 287 & $272-16$ & $229-1$ & 0 & 0 & $36: 0$ & $\ldots$ & $\ldots$ \\
\hline 289 & $272-8$ & $272-21$ & $2-4$ & $2-4$ & $0: 35$ & $\ldots$ & $\ldots$ \\
\hline 290 & $272-11$ & $272-21$ & $2-4$ & $2-4$ & $0: 14$ & $\ldots$ & $\ldots$ \\
\hline \multicolumn{8}{|c|}{ Segregation on } \\
\hline \multicolumn{8}{|c|}{ Cv. Katy } \\
\hline 292 & $225-1$ & $272-21$ & $0-1$ & $2-4$ & $14: 7$ & 2.33 & 0.78 \\
\hline 293 & $226-10$ & $272-21$ & $0-2$ & $2-4$ & $9: 5$ & 1.14 & 0.86 \\
\hline 294 & $272-17$ & $272-21$ & 0 & $2-4$ & $11: 17$ & 1.29 & $19.05^{* * *}$ \\
\hline 295 & $272-24$ & $272-21$ & $1-2$ & $2-4$ & $13: 18$ & 0.81 & $18.08 * *$ \\
\hline 296 & $272-8$ & $225-1$ & $2-4$ & $0-1$ & $26: 7$ & $10.94 * *$ & 0.25 \\
\hline 298 & $225-9$ & $272-21$ & $0-1$ & $2-4$ & $10: 9$ & 0.05 & $5.07 *$ \\
\hline 299 & $272-8$ & $225-16$ & $2-4$ & 1 & $19: 15$ & 0.47 & $6.63 *$ \\
\hline 300 & $272-8$ & $225-18$ & $2-4$ & 1 & $20: 6$ & $7.54 * *$ & 0.05 \\
\hline
\end{tabular}

a ITs ranging from 0 to 2 were considered avirulent, and ITs ranging from 2 to 4 were considered virulent.

b * indicates $P<0.05$; ** indicates $P<0.01$.

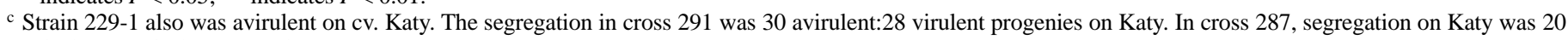
avirulent: 16 virulent progenies. 
26 has an avirulence in common with $340-2$ or that the avirulence genes in 294-26 and 295-31 are tightly linked to the $P 12$ gene in 340-2.

\section{DISCUSSION}

The cross between a rice field isolate from Texas and a fertile laboratory strain of $M$. grisea, cross 85 , gave an avirulent/virulent progeny ratio that suggested at least two genes condition avirulence on cvs. Lemont, Lebonnet, and Newbonnet (there was cosegregation on these three cultivars), two genes condition avirulence on cv. Leah, and two genes, or likely more, condition avirulence on cv. Katy. Four progenies from cross 85 that were avirulent on all five cultivars were used in backcrosses in an effort to establish strains that had single avirulence genes for one or more cultivars. The results from these $\mathrm{BC} 1$ progenies suggest two genes still segregated for avirulence on Lemont, Lebonnet and Newbonnet, one gene segregated for avirulence on Leah, and one, two, or more genes segregated for avirulence on Katy. After two additional generations of backcrossing to 70-6, some segregation ratios suggest that a single gene for avirulence is present in some BC2 and BC3 strains. (Crosses 223, 224, 225, 274, and 276 on cvs. Newbonnet, Lemont and Lebonnet [Table 1]; crosses 223, 225, and 274 on cv. Leah [Table 2]; and crosses 272, 273, 274, and 276 on cv. Katy [Table 3]). However, in the past $(2,3)$ we have found that a segregation ratio alone is not adequate to conclude that a single gene is present in a strain. Genetic interactions between different loci can affect the common phenotype, namely avirulence/virulence on a given cultivar. More test crosses are needed to confirm the result.

The type of test cross performed is illustrated with descendants of cross 272 . Cross 272 produced 20 avirulent and 10 virulent progenies on cvs. Newbonnet, Lemont, and Lebonnet. This segregation ratio is consistent with expectations of either one or two genes for avirulence on these cultivars (Table 1). Both parents in cross 272 were virulent on cv. Leah, and all progenies from cross 272 were virulent on Leah (Table 2). Cross 272 produced 16 avirulent and 14 virulent progenies on cv. Katy. This result is consistent with the segregation of a single gene for avirulence on Katy in strain 226-9 (Table 3). The progenies from cross 272 chosen for further test crossing were either avirulent on cvs. Newbonnet, Lemont, and Lebonnet and virulent on cv. Katy or vice versa.

Crosses between two progenies, both of which were avirulent on Newbonnet, Lemont, and Lebonnet (crosses 283 and 284), gave only avirulent progenies (Table 4). The conclusion from these two crosses is that 272-16 has one or more avirulence genes in common with 272-23 and 272-29. Crosses between progenies of cross 272 , both of which were virulent on Newbonnet, Lemont, and Lebonnet (crosses 289 and 290), gave only virulent progenies. We conclude that strains $272-8,272-11$, and 272-21 contain no suppressed avirulence genes. Strains 272-11 and 272-21 were of different mating types and were used in crosses with siblings that were avirulent on Newbonnet, Lemont, and Lebonnet (crosses 282, 286, and 288). The segregation ratios observed in crosses 286 and 288 suggest that a single gene is segregating for avirulence on these cultivars. The segregation ratio in cross 282 suggests that two genes for avirulence are segregating. This is expected if two genes, not one, for avirulence on these three cultivars were segregating in cross 272 (Table 1).

Crosses 294 and 295 involve crossing two strains, 272-17 and 272-24, both of which are avirulent on cv. Katy, with 272-21, which is virulent on Katy (Table 4). Cross 294 gave a progeny segregation ratio of 11 avirulent: 17 virulent that is consistent with one gene for avirulence coming from 272-17. Is this the correct interpretation? If it is correct, then intercrosses between progenies from cross 294 that are avirulent on cv. Katy should give only avirulent progenies. The cross $294-26 \times 294-21$ gave 47 progenies, all of which were avirulent on Katy. The conclusion is that 294-6 and 294-21 have a gene for avirulence on Katy in common. However, the cross $294-22 \times 294-21$ gave 11 avirulent and 20 virulent progenies on cv. Katy. Clearly strains 294-22 and 294-21 do not have the same genes for avirulence on Katy. The high proportion of virulent progenies is similar to the observations of a previous study in which a 26 avirulent: 28 virulent segregation ratio on cv. Katy was controlled by four genes: two avirulence genes and two specific suppressors of the avirulence genes (a 7:9 segregation instead of a 1:1 segregation) (3).

The segregation observed in cross 295, 13 avirulent: 18 virulent, also is consistent with the segregation of a single gene for avirulence on cv. Katy coming from 272-24 (Table 4). If the interpretation is correct, then intercrossing avirulent progenies from cross 295 should give only avirulent progenies. Crossing of 295-31 with 295-1 gave 75 progenies that were avirulent and 3 progenies that were virulent on cv. Katy (Fig. 1). The recovery of virulent progenies shows that the two strains, 295-31 and 295-1, are avirulent based on different genes that appear linked. The observed segregation ratio of approximately $1: 1$ in cross 295 is misleading concerning the number of genes segregating.

Crosses involving the progenies from cross 225 (Table 4) are consistent with the segregation of at least two genes for avirulence from 88-26 (Table 3). Although the number of progenies from cross 225 is small, the interpretation of the segregation of at least two genes for avirulence on Katy is supported by the test crosses between the progenies. In cross 292, 225-1 is avirulent and 272-21 is virulent on cv. Katy. The 14 avirulent: 7 virulent progeny ratio is consistent with both a $1: 1$ and a 3:1 segregation ratio (Table 4). Intercrosses of avirulent progenies 292-11 and 292-4 gave virulent progenies (Fig. 1). Cross 292 segregated for two genes. The same argument can be made for cross 293, which also segregated for two genes controlling avirulence/virulence on Katy.

The data presented in Figure 1 illustrate the difficulties in identifying the individual genes in the pathogen that control avirulence/virulence on a single cultivar, Katy. Previous studies $(2,3)$ have shown that cultivar specificity is always controlled by two interacting loci. A cross between two strains, one avirulent and the other virulent on a given cultivar, may give a one-gene segregation ratio (e.g., 1 avirulent:1 virulent), but this ratio can be obtained several ways. For example, a 1 avirulent: 1 virulent progeny ratio can result from crossing two avirulent strains with genotypes $P a s a \times p a s a$ or $P a s a \times P a S a$. The first cross segregates at the $P a$ avirulence locus, whereas the second cross segregates at the $S a$ suppressor locus. A 3 avirulent: 1 virulent progeny ratio can result from crossing two avirulent strains with genotypes $P a s a \mathrm{pb}$ $s b \times p a s a \mathrm{~Pb} s b$ or $\mathrm{Pa} s a \mathrm{~Pb} \mathrm{Sb} \times \mathrm{Pa} \mathrm{SaPb} s b$. The first cross segregates at the $P a$ and $P b$ avirulence loci, and the second cross segregates at the $S a$ and $S b$ suppressor loci. Although the data from backcrossing to 70-6 for several generations suggest that one or a few genes for avirulence/virulence on a given cultivar segregate in the $\mathrm{BC} 2$ and $\mathrm{BC} 3$ progenies, the intercrosses between strains avirulent on cv. Katy (Fig. 1) show that several genes segregate, that both $P$ and $S$ genes segregate, and that some genes are linked.

Strain $340-2$ is avirulent on cv. Katy based on genes P12 s12 (K. Akutsu and A. H. Ellingboe, unpublished data). Strain 340-2, which is mating type MAT1-1, was crossed with the six MAT1-2 strains (also avirulent on Katy) shown in Figure 1. In five of the six crosses, virulent progenies were given. In five of the six MAT1-2 strains, the genes for avirulence on Katy were different from P12 $s 12$. Strain 294-26 either has the same gene(s) for avirulence on Katy as 340-2, or the genes are tightly linked.

Many isolates of $M$. grisea have been obtained from rice fields in the United States. Many of these isolates have been inoculated on the international rice differentials to determine which physiological race each isolate represents. The diversity of the U.S. $M$. grisea population also has been studied by examining patterns of dispersed repeated DNA sequences (5). Both analyses suggest that the $M$. grisea population is not particularly diverse in the United 
States. It should be very useful to breeding programs to know which genes in each of the prevalent races, or molecularly identified groups, give avirulence on cv. Katy and on other host lines that are used as sources of resistance in breeding programs. The establishment of a set of strains each of which has a different, single gene for avirulence on cv. Katy (or on other host lines) will permit monitoring of the individual genes for resistance transferred to any new cultivars. A new cultivar that is resistant based on one to three or more genes may not change the resistance phenotype. However, a new cultivar that is resistant based on three genes, each of which is effective against all strains of the pathogen, is more likely to give longer lasting resistance than a new cultivar that has only one gene for resistance that is effective against all strains of the pathogen. The ability to monitor the interaction between the host and the pathogen one gene at a time is much more informative than monitoring race change on a set of differentials that may not have any genes in common with the sources of resistance used in breeding programs.

\section{LITERATURE CITED}

1. Chao, C. T., and Ellingboe, A. H. 1991. Selection for mating competence in Magnaporthe grisea pathogenic to rice. Can. J. Bot. 69:2130-2134.
2. Ellingboe, A. H. 1992. Segregation of avirulence/virulence on three rice cultivars in 16 crosses of Magnaporthe grisea. Phytopathology 82:597-601.

3. Lau, G. W., Chao, C. T., and Ellingboe, A. H. 1993. Interaction of genes controlling avirulence/virulence of Magnaporthe grisea on rice cultivar Katy. Phytopathology 83:375-382.

4. Leung, H. 1984. Genetic and cytological characterization of the rice blast fungus, Pyricularia oryzae Cavara. Ph.D. thesis. University of Wisconsin, Madison.

5. Levy, M., Romao, J., Marchetti, M. A., and Hamer, J. E. 1991. DNA fingerprinting with a dispersed repeated sequence resolves pathotype diversity in the rice blast fungus. Plant Cell 3:95-102.

6. Marchetti, M. A., Rush, M. C., and Hunter, W. E. 1976. Current status of rice blast in the southern United States. Plant Dis. Rep. 60:721-725.

7. Ou, S. H. 1980. A look at worldwide rice blast disease control. Plant Dis. 64:439-445.

8. Ou, S. H. 1980. Pathogen variability and host resistance in rice blast disease. Annu. Rev. Phytopathol. 18:167-187.

9. Ou, S. H. 1985. Rice Disease. 2nd ed. Commonwealth Mycological Institute, Kew, England.

10. Rossman, A. Y., Howard, R. J., and Valent, B. 1990. Pyricularia grisea, the correct name for the rice blast disease fungus. Mycologia 82:509-512.

11. Valent, B., Crawford, M. S., Weaver, C. G., and Chumley, F. G. 1986. Genetic studies of fertility and pathogenicity in Magnaporthe grisea (Pyricularia oryzae). Iowa State J. Res. 60:569-594.

12. Wu, B.-C. 1990. Studies on the mechanism of variation in Pyricularia oryzae (Magnaporthe grisea). Ph.D. thesis. Texas A\&M University, College Station. 